LUIS GONZÁLEZ

\title{
La diáspora de los intelectuales
}

Crisis en la investigación científica mexicana

F $N$ los años setenta del presente siglo se hacen muy I. notorias dos series de estorbos a la investigación humanística en México, concentrada, en sus cuatro quintas partes, en la capital de la República. Una de las series la constituyen los excesos de la investigación metropolitana y la otra, las deficiencias de los chisguetes de investigación en distintos lugares del pais. Dan cuerpo a la serie mayor los problemas del gigantismo, la burocracia excesiva, la esclerosis galopante, la fuga de cerebros hacia la política, la neurosis, la ideología en turno y la jerigonza. A la serie provinciana concurren poco oficio, pobreza, exceso de erudición, enanismo, falta de eco y esclavitud docente.

La estructura agigantada de casi todos los centros de investigación científica de la metrópoli se nota en el número tan crecido de sus investigadores verdaderos, en el albergue que da a simples parásitos de la labor académica y en lo desmesurado del personal administrativo. Por investigaciones recientes se sabe que en un instituto se estorban unos a otros más de medio centenar de científicos, en vez de ayudarse. De otro lado, los que se cuelan, y no saben o no les gusta investigar, contribuyen a la gordura de las instituciones. Peor sucede con los burócratas. Sabios, seudosabios y administrativos agigantan el tamaño de las comunidades académicas capitalinas. Además, los parásitos y los burócratas contribuyen también a los fenómenos de la esclerosis y la evasión o fuga de cerebros. 
La esclerosis de la gran mayoría de los centros de investigación metropolitanos suele manifestarse en la escasa producción de libros y artículos (o sea, en el caminar con lentitud) y en la sobredosis de personal de apoyo (es decir, el moverse con pies ajenos). Las publicaciones y su repercusión en el ámbito de la cultura son por ahora los mejores útiles para medir la agilidad de un instituto de ciencias del hombre. Si se aplican estas medidas a los institutos metropolitanos de México muy pocos resultan medianamente ágiles. La gran mayoría denota reumatismo paralizante muy avanzado, produce poco y de poca valía porque no se puede repicar y andar en la procesión al mismo tiempo.

Se advierte en no pocos institutos capitalinos dedicados a la investigación humanística una tendencia a evadirse, a fugarse de sus funciones propias para ir en ayuda del sector gubernamental, para obtener buenos recursos a cambio de servicios especiales y para hacerse de una imagen prestigiosa. En la ciudad de México se juntan los tres peligros mayores de toda labor cientifica: el poder, el dinero y la fama. La ciencia pierde muchas horas hábiles de los científicos porque se dejan tentar por las tareas administrativas, los puestos de rector y director de instituciones culturales, la buena vida, los empleos bien pagados, el ir de una reunión de cultos a otra, el andar de entrelucido, las comparecencias en televisión, el hacerse del micrófono, el recibir honores y el ser el centro del sarao.

Por otra parte, la región menos transparente del aire, la que ya no reconocería como suya José María Velasco, es poco recomendable para una buena ejecutoria de paisajistas y de investigadores. La neurosis de muchos científicos, atribuida al neblumo, la sonoridad mecánica, los itinerarios largos y lentísimos, el juntismo, la comititis, las excesivas obligaciones docentes y de otra índole y la falta de corazón de la capital, es obstáculo para la hechura de obras científicas en la cazuela capitalina. Cada vez es más el número de defeños convencidos de que se puede vivir con los nervios de punta y sin alegría con muchos transportes, espectáculos fabulosos y millones de seres humanos de mirada torva.

La búsqueda de la verdad ha dejado de ser el principio rector de muchos investigadores de la monstruosa metrópoli. 
En vez de preocuparse por el conocimiento del hombre, numerosos científicos sociales de la capital se preocupan por no ser tildados de agentes de la reacción y del imperialismo, por mantenerse al día en sistema ideológico-político o por el ansia de cantar lo que el grupo dominante desea oír. Se buscan los efectos de lo dicho por uno en los colegas y en los patrocinadores y no las aportaciones al conocimiento. Cada vez con mayor frecuencia se sustituye la curiosidad científica por las ideologías de moda en el poder y en la oposición. En forma creciente se evita desagradar a los poderosos o a sus enemigos en turno. Se cae en la propaganda y se margina la desnuda verdad.

Por último, el discurso ininteligible pretende hacer a un lado a la comunicación efectiva. El cantinflismo pedante, el uso de un vocabulario seudocientífico, la repetición de términos como concientizar, acomplejado coyuntural, mentalidades, coalescencia, encapsulación étnica, conducción, societario, involucionado, grupal, necesidad, logro, intelectual orgánico, estructura orgánica, audiotáctil, alienación, promocionarse, verticalidad, etnicidad y otros por el estilo han llevado a muchos estudiosos del hombre residentes en la ciudad de México a los confines de la torre de Babel. El buen humor en el decir, la prueba de no padecer deficiencias de lenguaje, tiende a esfumarse en el panorama metropolitano de la ciencia.

Las casas de investigación en provincia pecan generalmente de lo contrario de las capitalinas. Casi todas padecen enanismo. Según los investigadores de la investigación cientifica, con menos de veinticinco sabios ninguna comunidad académica puede tener un desarrollo saludable, y la mayoría de las pocas comunas provincianas están lejos de reunir ese minimo de miembros. Como si eso fuera poco, los dedicados a la creación científica fuera de la metrópoli suelen arrear cursos en los niveles de preparatoria y licenciatura a mañana, tarde y noche. Por lo demás, la mies provinciana es mucha para muy pocos y distraídos operarios. Al revés de la capital, donde se vuelve repetidas veces a los mismos asuntos, en la multitud de cuauhtitlanes de México, en los multiméxicos, hay abundantes problemas y temas en busca de estudioso y autor; hay escasez de investigadores científicos y abundancia de cuestiones vírge- 
nes. Fuera de México capital casi todo está por conocerse del México múltiple.

En provincia, la riqueza de la realidad suele hacer menos necesarios los cimientos ideológicos, quedarse en la simple erudición, en el almacenamiento de datos sin sentido, en la hechura de tratados de todas las cosas y algunas más. Los investigadores extrametropolitanos caen frecuentemente en el pecado de la omniciencia, quizá por escasez de oficio. Los institutos científicos de la capital alojan doctores; los de provincia, aficionados. El poco oficio, la escasez de formación profesional, la proximidad a la brujería acompaña a menudo a los investigadores que trabajan y le dan vuelo a la imaginación fuera del Distrito Federal. Muchos sabios de provincia se quejan con justa razón de vivir en el dilema de cuando hay tiempo no hay pan y cuando consiguen para vivir no disponen de horas para ponerse en mangas de investigador. Aparte de pobreza económica padecen de pobreza cultural. Los archivos y las bibliotecas se juntan en una metrópoli cuyos sabios rehuyen la lectura y siguen ausentes en los sitios donde existen los aspirantes a ratones de biblioteca. Fuera de México se investiga poco en papeles, en horas perdidas, sin estímulos económicos y sin el aliciente de la repercusión o la fama. Según Bertrand Russell, "un cierto grado de soledad en espacio y tiempo es indispensable para producir la independencia necesaria que requiere un trabajo serio", pero el exceso de soledad y la falta de estímulo no ayudan a ningún sabio.

\section{Alternativas para salir de la crisis}

Con el propósito de corregir las deformaciones de la investigación científica en México metrópoli y en México nación se han propuesto y aún puesto en marcha no pocas políticas. A principios de los setenta se funda el Consejo Nacional de Ciencia y Tecnología para promover la obra de los científicos mexicanos. A partir de entonces proliferan, con la ayuda del poderoso, los programas de investigación y las escuelas formadoras de investigadores, y se aplica la técnica japonesa de enviar becados a universidades de los países del primer mundo para cosechar rápidamente buenos científicos. También aumentan los 
premios para la creación científica, nace vigoroso el Sistema Nacional de Investigadores y un número creciente de revistas y libros da a conocer los frutos de cada vez más practicantes de las ciencias físico-matemáticas, biomédicas y humanísticas. Los gastos de CONACYT y SEP para el desarrollo de ciencia y tecnología suben $40 \%$ anualmente entre 1976 y 1981.

El único mecenas de la investigación científica ha sido el gobierno emanado - como se dice en los discursos oficialesde la Revolución Mexicana. Casi siempre el mecenas oficial es consciente de dónde le aprieta el zapato a la investigación científica de aquí y ahora. En los últimos años se ha vuelto, por añadidura, liberal en el patrocinio de diagnósticos y de planes de reforma. La organización de coloquios organizados por la Secretaría de Programación y Presupuesto, la Secretaría de Educación Pública y el Consejo Nacional de Ciencia y Tecnología demuestran el interés de las autoridades en el buen desarrollo de las ciencias humanas en México. En esos coloquios se han propuesto distintas curas para conseguir la salud de la investigación científico-humanística: dotar de suficientes recursos económicos a las instituciones investigadoras, enviar jóvenes investigadores a las universidades de los países del primer mundo, hacer un plan nacional de política científica, desarrollo de grupos institucionales, unir a los distintos mecenas de las labores culturales y descentralizar los institutos encargados del fomento científico. De los múltiples remedios propuestos, sólo trataré del último. Sólo a esta cura, puesta de moda por la calamidad del 19 de septiembre de 1985, se referirá mi ensayo. Acerca de los demás remedios debe consultarse Sísifo y Penélope, una obra muy seria y regocijada del doctor Ruy Pérez Tamayo.

Con el propósito de convertir a México en un país productor de conocimientos científicos y tecnológicos, desde finales de los setenta, la rectoría del Estado ha mirado con buenos ojos las propuestas encaminadas a sacar del D.F. a los investigadores. En las juntas de la Asociación Nacional de Universidades y en otros foros, patrocinados por la Secretaría de Educación Pública y el Consejo Nacional de Ciencia y Tecnología, se ha propuesto una y otra vez la descentralización de la investigación científica como el medio más adecuado para un buen desarrollo intelectual del país. Todas las propuestas coinciden 
en el diagnóstico, pero no en los métodos a seguir. Algunos proponentes ven la solución en que los institutos universitarios de la capital pongan sucursales en la provincia mexicana. Otros recomiendan dar ayuda económica y académica a los institutos satélites de las universidades de los Estados. Unos terceros proponen la apertura de institutos autónomos de investigación científica en la vasta geografía del país.

Los tres modos de descentralización recomendados han recibido aceptaciones y repudios. La apertura de sucursales de la UNAM, la UAM, el Politécnico y otros institutos metropolitanos de educación superior parece ser la manera más factible y que ofrece mayores garantías (ISSSTE y esas cosas) a los descentralizados. Como quiera, sus oponentes dicen: "Las sucursales acabarán por convertirse pronto en siberias, en campo de destierro para investigadores conflictivos o de poco fuste de las universidades metropolitanas". ¿Quién no usará de esa magnífica ocasión para desparasitarse? Tampoco resulta caro ni difícil poner investigadores, con todo y su torta, en los institutos de investigación existentes en las universidades foráneas. En este caso, se aprovecha una estructura administrativa en funciones. Sin embargo, no falta quien argumente que con tal procedimiento no se consigue enderezar los muy torcidos troncos de la exigua investigación provinciana. Según esta opinión, los que se van de redentores a las universidades extrametropolitanas terminan crucificados. La investigación local aficionada se vuelve iracunda contra las ínfulas de los sabios que envía la metrópoli. Por lo mismo, los dispuestos a disminuirse ante la opinión pública por salir a trabajar fuera de la metrópoli deben hacer sus propios institutos, sus caparazones académicos independientes, aunque tampoco éstos, según los experimentos, aseguran una descentralización sólida y duradera. Toda planta nueva es muy vulnerable en México. Se prefiere la veredita conocida al ancho camino por conocer.

Sin embargo, se han ensayado las tres vías. Todo lo propuesto se ha puesto en práctica. La UNAM y el Politécnico han instalado algunas casas de ciencia y tecnología en diferentes lugares del pais. SEP, CONACYT y las instituciones de cultura superior de la capital han contribuido con recursos humanos y económicos a la apertura y consolidación de programas para 
hacer investigadores e investigaciones cientificas en las universidades provincianas. En 1978, la UNAM lanza un ambicioso proyecto de desconcentración; crea estaciones foráneas y sucursales que aspiran a ser permanentes. No todas las aventuras unamitas han levantado cabeza; su lento desarrollo ha sido poco saludable. Varias padecen de los males anunciados. Con todo, aún no es tiempo de opinar. Como dice Ruy Pérez Tamayo, los frutos de las experiencias aludidas no pueden cosecharse de un día para otro. La hechura "de una tradición científica es obra no sólo del talento sino también del tiempo". Aún no se puede decir la última palabra sobre las sucursales de los centros universitarios en provincia o sobre el fomento de institutos dependientes de las universidades provincianas.

Sin embargo, ya se pueden hacer comerciales del tercer modelo de descentralización. Ya hay frutos en el mercado del submodelo consistente en abrir colegios estatales a imagen y semejanza del Colegio de México, pero no dependientes de la matriz. A partir de 1978 se pone en marcha la nueva política, se inicia la experimentación con el submodelo Colmex. A principios de 1979 se abre El Colegio de Michoacán en Zamora. A un trienio de distancia de Colmich, como le decimos al de Michoacán privadamente, brotaron otros cuatro planteles similares al de Zamora. En 1982, arrancan El Colegio de Sonora, El Colegio del Bajío, El Colegio de la Frontera Norte y El Colegio de Jalisco. Enseguida empiezan los sustos acarreados por la crisis que ponen un hasta aquí a la racha de fundaciones, quizá indebidamente por lo que diré enseguida. Me voy a permitir reseñar, en pocas palabras la vida del primer colegio estilo Colmex, la trayectoria de un proyecto de descentralización sin antecedentes en México, pero ya exitoso. Pido mil palabras para referir la fundación y discurso del Colegio de Michoacán.

\section{La alternativa que ya probó su eficacia}

Después de la aprobación de un diseño de instituto por la asamblea de socios fundadores; es decir, por la SEP, CONACYT, El Colegio de México, CIESAS, el gobierno de Michoacán y la Universidad Michoacana, se propuso abrir el instituto en Zamora, a sabiendas de que en Morelia están los me- 
jores archivos del Estado, se prodigan espectáculos culturales y existen fondos bibliotecarios del mayor interés. Se rechazó a la capital michoacana por su cercanía al sol de la gobernatura y a los parientes de la universidad. San gobierno y san Nicolás podian chuparse en un santiamén a la criatura colmichiana.Quizá Zamora no haya sido el lugar michoacano más a propósito para establecer el Colmich, pero es notable por su cercanía a Guadalajara, Morelia y la capital guanajuatense y por ser buen campo de estudio. Quizá quepa quejarse del clima tibio, el sinfín de insectos, las amibas y lo difícil de conseguir casas en renta y de hacerse de un centro de información adecuado.

El Colegio de Michoacán nació seguro de que sin libros, revistas, papeles y mapas hay pocas posibilidades de investigación. Por lo mismo, una de sus primeras miras fue la de hacerse de un acervo importante de impresos. Se descartó la idea de una biblioteca con un poquito de todo, así como la de hacer un depósito de libros de texto y similares. Se pensó que lo más acorde a los fines del instituto sería una biblioteca especializada en obras de ciencias sociales y en publicaciones relativas al Occidente de México. En su primer cuatrenio de vida Colmich logró reunir 23000 volúmenes de libros y 25000 de revistas. Ahora, después de ocho años de fundado, dispone de casi cien mil volúmenes de libros y revistas, siete mil mapas, varios archivos particulares, una buena colección de fotografías y otra de grabaciones sonoras y videocartuchos.

Por lo que mira a equipo, también se va de prisa. Se cuenta ya con un piano y otros utensilios de los viejos tiempos y con buen número de cámaras fotográficas, microcomputadoras, videograbadoras, una composer y otros artefactos de la tecnología moderna. Por otra parte, hay un breve personal de apoyo lo más distante posible de la burocracia kafkiana. Se partió de la idea de que los científicos no necesitan jefes, pues saben lo que desean, pero sí ayudantes para las cosas prácticas, pues suelen ser torpes en la resolución de problemas nimios. Con todo, el personal de apoyo del Colegio nunca ha superado en número al personal académico, y en definitiva, no ha caído en la tentación de la abundancia de ayudantes que casi siempre desemboca en la dictadura de éstos sobre los ayudados.

En casonas alquiladas, mientras se termına su edificio en 
construcción; con una biblioteca ya respetable y en edificio ad hoc; con equipo que cubre satisfactoriamente los requerimientos de contabilidad, transporte, edición de libros y otros menores; con un presidente que es ahora el conocido y respetado historiador Andrés Lira y con una comunidad académica que comenzó siendo de ocho responsables de programas de estudio y ahora es de treinta residentes, un tercio de los cuales es del Sistema Nacional de Investigadores, El Colegio de Michoacán ha cumplido con sus fines más allá de lo esperado, y según acaba de decirlo FLACSO después de una vasta exploración, cumple más cabalmente que cualquier centro capitalino en la tarea de hacer investigadores.

Hasta ahora la meta mayor de investigar se ha constreñido al campo de las ciencias sociales y las humanidades. Se ha puesto el ojo en la sociedad michoacana y jalisciense, aunque no en exclusiva. Se ha preferido el estudio de los problemas palpitantes, pero sin restar importancia a los estudios históricos. Aunque se hayan fomentado especialmente las investigaciones en equipo y multidisciplinarias no se les ha hecho el feo a las búsquedas individuales ni a las muy eruditas. Aunque los temas a investigar han nacido generalmente de la voluntad de los investigadores, se ha colaborado en investigaciones propuestas por algunos socios. Este es el caso de las monografías estatales para la educación primaria, las historias de Colima y de Michoacán y las investigaciones acerca de la lucha por la independencia de México en la zona occidental de la República. Por otra parte, cada uno de los cuatro centros en que se divide El Colegio de Michoacán ha instituido métodos propios y aun originales de cumplir con sus funciones dentro de los campos de la historia, la antropología social, los estudios rurales y el estudio de las tradiciones.

Tres de los centros forman gente apta para la investigación. El Colegio de Michoacán da cursos, orienta en prácticas de campo y dirige tesis para optar a tres maestrías y está por poner en marcha un doctorado en ciencias sociales. Desde 1979 se adiestra en la investigación y la escritura de obras científicas a grupos pequeños de estudiantes. De los quince de la primera hornada, once han defendido tesis bien hechas sobre temas originales y jugosos. Los grupos de las tres siguientes 
promociones han superado en número a la primera y la que recibe ahora cursos lo dobla. Los egresados no han tenido dificultad para acomodarse en los centros de investigación del país, y por lo menos un par, es ahora parte académica del Colmich.

Nuestro instituto se enorgullece por haber formado ya medio centenar de investigadores, pero sin duda su orgullo mayor se cifra en la enorme cosecha de libros y artículos resultantes de las labores inquisitivas de su planta de investigadores. Con todo, su meta de difusión no se agota en el terreno editorial. Auspicia desde 1981 un cine club; ofrece conferencias públicas, a razón de una por semana; organiza anualmente congresos de sabios alrededor de un tema de interés nacional. Los ocho congresos tenidos hasta ahora han logrado cierto prestigio. Por último, sus investigadores asisten algunas veces a asambleas sabias que tienen lugar en México y en el extranjero, y también desempeñan el papel de profesores visitantes fuera de la sede zamorana.

Los académicos colmichianos publican libros y artículos que superan con mucho la producción bibliográfica de los institutos de la capital de la República. Quizá por la escasez de distracciones en la pequeña ciudad de Zamora, los miembros de Colmich se divierten escribiendo a raudales. En siete años y medios han publicado un centenar de libros, una docena de folletos y doscientos sesenta y siete artículos largos y de fondo. De la producción libresca, aproximadamente el $65 \%$ pertenece al rubro de publicaciones científicas; el 20\%, a la categoría de publicaciones didácticas y el $15 \%$ restante son libros de divulgación. Al número de los cien volúmenes publicados deben agregarse doce en prensa o en vísperas de imprimirse. En suma, en promedio el investigador colmichiano produce un libro cada veinte meses y un artículo de fondo cada seis. La calidad de estas obras es buena; no desluce ante los frutos de las ciencias sociales capitalinas y a veces los aventaja. El Colegio de Michoacán es cuatro veces más fecundo que la más fecunda de las instituciones metropolitanas. Nuestros informes de investigación resultan mucho más baratos que los producidos en la metrópoli.

Si de los primeros volúmenes de los colmichianos pudo 
decirse que eran reflejo de investigaciones previas a su incorporación al Colegio y que no se ocupaban de problemas del Occidente del país. De los últimos cabe decir que se ocupan de asuntos tan locales como las formas de poder en la tierra fría de Michoacán, la agricultura en la tierra caliente, el comercio de Nahuátzen, las relaciones entre religión y capitalismo en el Bajio Zamorano, los obreros de Río Grande, la industria de Guadalajara, la alfarería de Chilchota, los braceros de Gómez Farías y otros temas similares. Sin lugar a dudas El Colegio de Michoacán ya contribuye seria y asiduamente al conocimiento de la zona occidental de México y quizá contribuya de este modo a la solución de los problemas occidentales.

\section{Diez moralejas descentralizadoras}

Lo expuesto anteriormente en forma sucinta y no cabal permite extraer varias moralejas útiles para la descentralización de los nidos de las ciencias del hombre que hoy dormitan y sufren morbos, taras y neurosis en la hidrocefálica ciudad de México.

1. Los primeros siete años de vida del Colegio de Michoacán demuestran, que no obstante el arraigado centralismo de quienes pagan y disponen, son posibles y deseables los institutos pequeños y libres dedicados a la investigación científicosocial y a la formación de investigadores fuera y lejos de la cabezota de la República Mexicana. Ciertamente no todo es todavía miel sobre hojuelas. Andrés Lira, líder de Colmich, se duele del "retraso en la autorización y pago" de los subsidios oficiales. Esto parece decir que aún no se ha conseguido la descentralización ideal para la buena marcha de los institutos provincianos.

2. Con todo, de la vida octonaria de Colmich se deduce la posibilidad de erigir un instituto científico de excelencia en un centro urbano de menos de cien mil habitantes, donde no hay universidad ni tampoco oficinas públicas de importancia como son las que presiden el gobernador y sus inmediatos colaboradores. Ya se puede establecer un buen número de buenos estudiosos, sin mengua de su prestigio, fuera de sedes universitarias y capitales políticas, en simples cabeceras de municipio ha- 
bitada por gente orgullosa de dar asilo a los sacerdotes del saber científico.

3. Todavía más. Parece preferible abrir los colegios provincianos estilo Colmex a buena distancia de las capitales de los Estados, algunas ya muy populosas, y todas con condiciones apropiadas para hacer una carrera política, para conseguir la caída de los intelectuales en la tentación del poder. Ya casi todas las metrópolis de los Estados cuentan con institutos de educación universitaria y cabe aventurar la idea de que los centros de investigación establecidos en provincia son más fecundos si laboran independientes, con pocos nexos y alejados de las universidades provincianas, por regla general revoltosas, y en todo caso sólo interesadas en la hechura de técnicos de la medicina, las leyes, la ingeniería, la contàbilidad y los negocios. La investigación no debe vincularse con la ensenanza de técnicos, que sí de investigadores.

4. La experiencia colmichiana parece demostrar que el estudio de las regiones desde el interior de ellas da muy buenos frutos, sin duda superiores a los producidos por antropólogos, sociólogos, politólogos y economistas adheridos tenazmente a la capital de la República. Puede afirmarse que se conseguirá el conocimiento científico de la heterogeneidad mexicana cuando se le analice desde miradores próximos a cada región y a cada terruño de un país de doscientas regiones y de más de dos mil municipios. El estudio insitu de cada una de las piezas del mosaico mexicano permitirá el conocimiento y quizá la salvación de ese mosaico.

5. En las ciudades pequeñas de la provincia mexicana hay pocos riesgos de contraer el gigantismo, la neurosis, la evación, el "rollo" y otros tumores que aquejan a los institutos de la capital. Está aún distante el día en que mucha gente quiera pertenecer a un colegio provinciano. También es dificil que una ciudad de cien mil habitantes logre destrozar los nervios en forma tan persistente como una urbe de quince millones. Tampoco es fácil obtener fuerza y ser arlequín en el carnaval de la propaganda en ciudades menores sin diarios de mucha circulación y sin emisoras de tele. Por último, en lugares donde la competición intelectual no puede ser mayúscula no se requiere el uso de jergas apantalladoras. 
6. En los tiempos que corren ya no se puede sostener el dicho de que a la provincia sólo se va a engordar y a embrutecer. Generalmente los alimentos son mejores que los capitalinos, pero también se ha puesto de moda allá el mantenerse esquelético al través de hambre y carreras. La escasez de centros de información, espectáculos, diversiones y otros serviciós ofrecidos fuera de México en forma muy raquítica o grotesca son fácilmente superables en este mundo tan tecnificado, sobre todo en lo que toca a medios de comunicación. Ya se pueden tener en los sitios más recónditos noticias tan frescas y tan copiosas como en la ciudad monstruo. Ya la televisión y el cine llevan a todos los rincones buenos espectáculos para divertirse, horrorizarse y disfrutar de la belleza. La experiencia ganada en Colmich asegura que en una ciudad pequeña de provincia se pueden disfrutar las dosis adecuadas de comunicación y aislamiento tanto para los investigadores maduros como para los estudiantes.

7. Si a un colegio provinciano se le dota de una buena planta de maestros y sólo admite alumnos en poca cantidad y becados, se transfigura necesariamente en un almácigo hacedor de jóvenes expertos en la investigación a fondo del comportamiento del hombre. La fórmula Colmex de descentralizar la vida académica parece ser el sistema menos costoso y más eficiente de hacer investigadores con estudiantes rigurosamente seleccionados, investigadores que arrancan hacia el estudio de por vida con buen entrenamiento en prácticas de campo, dos o tres artículos publicables y una tesis gorda, original, bien estructurada y mejor escrita. Sin duda, no existe ambiente más favorable al estudio y dedicación de los estudiantes que el recoleto de la provincia.

8. Queda claro, y es de suma importancia para ésta época de crisis, que las instituciones como El Colegio de Michoacán cuestan mucho menos y producen mucho más que sus equivalentes capitalinas. La infraestructura de los institutos humanísticos es menos cara que la de los centros de investigación biomédica. El costo del instrumental informativo que requiere un instituto de la marca Colmex no es muy elevado y es posible conseguirlo con muy poco si se consigue descentralizar algunos ejemplares de las bibliotecas capitalinas. Los gastos de 
mantenimiento de una biblioteca de reciente apertura bien equipada son mucho menores que los de una biblioteca añosa y muy atendida por numerosos bibliotecarios que cobran sueldos muy superiores a los de las computadoras. Por otra parte, es muchas veces más barato el hacer y publicar el fruto de una investigación (libro, artículo o videocartucho) en un colegio de provincia que en un instituto metropolitano.

9. Las solicitudes de incorporación al Colmich recibidas recientemente demuestran la voluntad descentralizadora de un número cuantioso de investigadores que ahora sí se manifiestan verdaderamente molestos por vivir en el Distrito Federal. Son gente bien formada, madura y productiva que hasta hace poco sólo de dientes para afuera expresaban el deseo de salirse de la ciudad más poblada del mundo y cada vez menos habitable. Quizá el terremoto del 19 de septiembre decidió la voluntad de salir de muchos que antes se debatían entre el me voy y no me voy. Quizá pasado el miedo del sismo las inclinaciones descentralizadoras cambien.

10. Esta parece una hora muy apropiada para extender los beneficios de la investigación científica a toda la sociedad nacional mediante el apoyo a los colegios de provincia ya existentes y la apertura sin prisas, bien planeada, de nuevos colegios de investigadores científicos que naturalmente, para funcionar bien, necesitan recibir subsidio bastante y oportuno, tener autonomía administrativa y académica y publicar sin cortapisas los resultados de sus investigaciones. La diáspora de los intelectuales dentro del territorio de la república será el punto básico de la avizorada revolución cultural de México. 